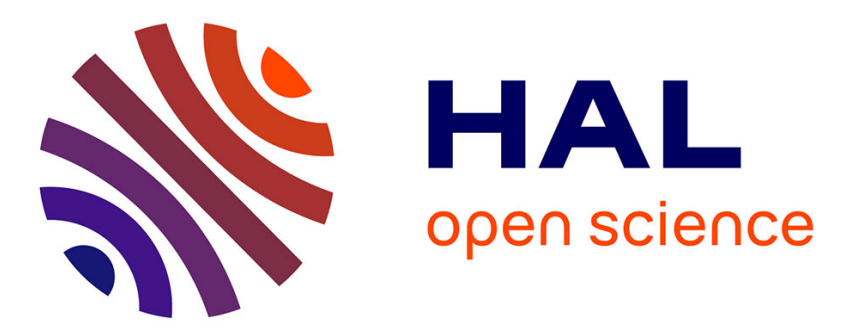

\title{
Structural uncertainty modeling for nonlinear geometric response using nonintrusive reduced order models
}

\author{
X. Q. Wang, Marc Mignolet, Christian Soize
}

\section{To cite this version:}

X. Q. Wang, Marc Mignolet, Christian Soize. Structural uncertainty modeling for nonlinear geometric response using nonintrusive reduced order models. Probabilistic Engineering Mechanics, 2020, 60 (103033), pp.1-9. 10.1016/j.probengmech.2020.103033 . hal-02468676

\section{HAL Id: hal-02468676 https://hal.science/hal-02468676}

Submitted on 6 Feb 2020

HAL is a multi-disciplinary open access archive for the deposit and dissemination of scientific research documents, whether they are published or not. The documents may come from teaching and research institutions in France or abroad, or from public or private research centers.
L'archive ouverte pluridisciplinaire HAL, est destinée au dépôt et à la diffusion de documents scientifiques de niveau recherche, publiés ou non, émanant des établissements d'enseignement et de recherche français ou étrangers, des laboratoires publics ou privés. 


\title{
STRUCTURAL UNCERTAINTY MODELING FOR NONLINEAR GEOMETRIC RESPONSE USING NONINTRUSIVE REDUCED ORDER MODELS
}

\author{
X.Q. Wang ${ }^{\mathrm{a}}$, Marc P. Mignolet ${ }^{\mathrm{a}}$, and Christian Soize ${ }^{\mathrm{b}}$ \\ ${ }^{\mathrm{a}}$ SEMTE Faculties of Mechanical and Aerospace Engineering, \\ Arizona State University, Tempe, AZ 85048, USA \\ xiaoquan.wang.1@asu.edu,marc.mignolet@asu.edu \\ ${ }^{\mathrm{b}}$ Laboratoire Modélisation et Simulation Multi Echelle, \\ Université Paris-Est Marne-la-Vallée, 77454 Marne-la-Vallee, France \\ christian.soize@u-pem.fr
}

\begin{abstract}
The focus of the present investigation is on the introduction of uncertainty directly in reduced order models of the nonlinear geometric response of structures following maximum entropy concepts. While the approach was formulated and preliminary validated in an earlier paper, its broad application to a variety of structures based on their finite element models from commercial software was impeded by two key challenges. The first of these involves an indeterminacy in the mapping of the nonlinear stiffness coefficients identified from the finite element model to those of the reduced order model form that is suitable for the uncertainty analysis. The second challenge is that a key matrix in the uncertainty modeling was expected to be positive definite but was numerically observed not to be. This latter issue is shown here to be rooted in differences in nonlinear finite element modeling between the commercial software and the theoretical developments. Both of these challenges are successfully resolved and applications examples are presented that confirm the broad applicability of the methodology.
\end{abstract}

KEY WORDS: Uncertainty modeling, maximum entropy, uncertain structure, nonlinear geometric structural response, reduced order modeling

\section{INTRODUCTION}

Over the last two decades, maximum entropy concepts have been broadly and very successfully used to model uncertainties in structures directly at the level of reduced order models (ROMs) constructed from the finite element model of the mean structure, see [1,2] for reviews. In addition to its capability to account for some epistemic uncertainty, this approach is computationally much more expedient than its alternative, which is to first introduce uncertainty in the finite element model then construct a ROM for each sample of the random structure. The computational saving is particularly significant for large size linear finite element models but also for nonlinear geometric problems where the ROMs include a large number of linear, quadratic, and cubic stiffness coefficients, see [3] for an overview. These coefficients must either be identified from a standard (e.g., commercial) finite element model using nonintrusive techniques [3-5] or computed using a dedicated finite element formulation [6-9] based on the reduced order modeling results of [10]. The latter of these two approaches lends itself naturally to the introduction of uncertainty at the ROM level using the maximum entropy concepts as originally discussed in [10]. The application of this strategy to the ROMs identified nonintrusively [3-5] from a commercial finite element code has been exemplified in [10] on a simple flat beam structure. More complex applications of this approach have been impeded by two key challenges, of decomposition and non-positive definiteness, in transforming the identified ROM into one that is suitable for the 
uncertainty analysis. The focus of the present investigation is on efficiently resolving these two challenges and applying them to a representative set of structures in the nonlinear geometric regimes.

\section{REDUCED ORDER MODELS OF NONLINEAR GEOMETRIC STRUCTURAL RESPONSE}

The reduced order models considered in the present study are based on a representation of the nonlinear geometric response of the structure in the form

$$
\underline{u}(t)=\sum_{n=1}^{M} q_{n}(t) \underline{U}^{(n)}
$$

where $\underline{u}(t)$ denotes the vector of displacements of the finite element degrees of freedom, $\psi^{(n)}$ are basis functions (modes) of the ROM specified in the spatial domain of the finite element mesh, and $q_{n}(t)$ are the time dependent generalized coordinates.

To obtain a set of nonlinear ordinary differential equations for the generalized coordinates $q_{n}(t)$, it is convenient to consider the continuum equivalent of Eq. (1), i.e.,

$$
u_{i}(\boldsymbol{X}, t)=\sum_{n=1}^{M} q_{n}(t) U_{i}^{(n)}(\boldsymbol{X}), i=1,2,3,
$$

where $X$ denotes an arbitrary point of the structure in its undeformed configuration. Then, introducing Eq. (2) in the equations of finite deformation elasticity and proceeding with a Galerkin approach provides the desired equations. This process was accomplished in [2] considering a Kirchhoff-Saint Venant type material in which the 2nd Piola-Kirchhoff stress tensor $S$ is linearly related to the Green strain tensor $E$ by

$$
S_{i j}=C_{i j k l} E_{k l}
$$

where $C$ denotes the deformation independent 4th order elasticity tensor. Under this material assumption, it was shown in [2] that the generalized coordinates $q_{n}(t)$ satisfy the equations

$$
M_{i j} \ddot{q}_{j}+D_{i j} \dot{q}_{j}+K_{i j}^{(1)} q_{j}+K_{i j l}^{(2)} q_{j} q_{l}+K_{i j l p}^{(3)} q_{j} q_{l} q_{p}=F_{i},
$$

where summation over repeated indices is implied. In the above equation, $M_{i j}$ denote the elements of the mass matrix, $K_{i j}^{(1)}, K_{i j l}^{(2)}, K_{i j l p}^{(3)}$ are the linear, quadratic, and cubic stiffness coefficients and $F_{i}$ are the modal forces. Note that a damping matrix $D$ was also introduced in Eq. (4) to model dissipation.

Note in Eq. (4) that a series of terms involve the same monomials of the generalized coordinates, e.g. $K_{i j l}^{(2)}$ and $K_{i l j}^{(2)}$, and thus these terms may naturally be regrouped leading to

$$
M_{i j} \ddot{q}_{j}+D_{i j} \dot{q}_{j}+K_{i j}^{(1)} q_{j}+\bar{K}_{i j l}^{(2)} q_{j} q_{l}+\bar{K}_{i j l p}^{(3)} q_{j} q_{l} q_{p}=F_{i},
$$

which is very similar to Eq. (4) except that there is no repetition in the monomials because $\bar{K}_{i j l}^{(2)}$ and $\bar{K}_{i j l p}^{(3)}$ are nonzero only for $j \leq l$ and $j \leq l \leq p$. Then, comparing Eqs (4) and (5) yields

and

$$
\bar{K}_{m n l}^{(2)}= \begin{cases}0 & \text { for } l<n \\ K_{m n n}^{(2)} & \text { for } l=n \\ K_{m n l}^{(2)}+K_{m l n}^{(2)} & \text { for } l>n\end{cases}
$$




$$
\bar{K}_{m n l p}^{(3)}=\left\{\begin{array}{lr}
0 & \text { unless } p \geq l \geq n \\
K_{m n n n}^{(3)} & \text { for } p=l=n \\
K_{m n l l}^{(3)}+K_{m l n l}^{(3)}+K_{m l l n}^{(3)} & \text { for } p=l>n \\
K_{m p l l}^{(3)}+K_{m l p l}^{(3)}+K_{m l l p}^{(3)} & \text { for } p>l=n \\
2 K_{m n l p}^{(3)}+2 K_{m p l n}^{(3)}+2 K_{m l p n}^{(3)} & \text { for } p>l>n
\end{array}\right.
$$

As discussed in [10], the symmetry properties of the elasticity tensor also imply that

$$
K_{m n l p}^{(3)}=K_{n m l p}^{(3)}=K_{m n p l}^{(3)}=K_{l p m n}^{(3)} \text {. }
$$

While Eqs (4) and (5) were derived based on the continuum representation of Eq. (2), they are assumed here to be valid as well for a discrete, finite element model. In fact, the identification of the coefficients $\bar{K}_{i j l}^{(2)}$ and $\bar{K}_{i j l p}^{(3)}$ from nonintrusive outputs of commercial finite element software (e.g., Nastran) has been studied and can be achieved in different ways, see discussions in [3-5], given the basis functions $\underline{\psi}^{(n)}$. The selection of these vectors is discussed in details in [7,9] and references therein and is not repeated here for brevity.

\section{MAXIMUM ENTROPY UNCERTAINTY MODELING AT THE ROM LEVEL}

The modeling of uncertainty at a ROM level has been developed, see [1,2], as a constrained optimization problem in which the entropy of the random parameters of the ROM is maximized under constraints which correspond to (i) physical requirements that these parameters must satisfy and (ii) conditions imposed by the user. To exemplify this strategy, consider the important case in which the ROM involves one or multiple positive definite symmetric matrices as occurs for example in linear structural dynamics. Let $\boldsymbol{A}$ be that random matrix and denote by $p_{A}(a)$ its probability density function which is defined over the domain of support $\bar{\Omega}$ such that $A$ is positive definite and symmetric, i.e., with

$$
A_{i j}=A_{j i} \text {. }
$$

Then, the entropy is

$$
S=-\int_{\bar{\Omega}} p_{A}(a) \ln p_{A}(a) d a
$$

where $p_{A}(a)$ must satisfy

$$
\int_{\bar{\Omega}} p_{A}(a) d a=1
$$

In addition to the physical requirements of symmetry and positive definiteness, it is also required that the mean of $\boldsymbol{A}$, denoted as $\overline{\boldsymbol{A}}$ is known, that is,

and moreover that

$$
\int_{\bar{\Omega}} a p_{A}(a) d a=\bar{A}
$$

$$
\int_{\bar{\Omega}} \ln [\operatorname{det}(a)] p_{A}(a) d a=v \text { finite }
$$


which guaranties that the inverse matrix $\boldsymbol{A}^{-1}$ of $\boldsymbol{A}$, which exists almost surely, is a second-order random variable (mean-square integrable).

The probability density function $p_{A}(a)$ maximizing $S$ given the constraints of Eqs (8),(10)-(12) can be derived by calculus of variation and is found to be

$$
p_{A}(a)=\tilde{C}[\operatorname{det}(a)]^{\lambda_{0}-1} \exp \left[-\operatorname{tr}\left(\tilde{\mu}^{T} a\right)\right]
$$

where $\tilde{C}$ is the appropriate constant to satisfy the normalization condition, Eq. (10) and $\mu$ and $\lambda_{0}$ are the Lagrange multipliers associated with the constraints of Eqs (11) and (12), respectively. After a change of random variables, it is found that the matrices $A$ of joint probability density function $p_{A}(a)$, Eq. (13), can be generated as

$$
\boldsymbol{A}=\overline{\boldsymbol{L}} \boldsymbol{H} \boldsymbol{H}^{T} \overline{\boldsymbol{L}}^{T}
$$

where $\overline{\boldsymbol{L}}$ is any decomposition, e.g., Cholesky, of $\overline{\boldsymbol{A}}$, i.e.,

$$
\overline{\boldsymbol{A}}=\overline{\boldsymbol{L}} \overline{\boldsymbol{L}}^{T} \text {. }
$$

Moreover, $\boldsymbol{H}$ is a lower triangular matrix such that (see also Fig. 1)

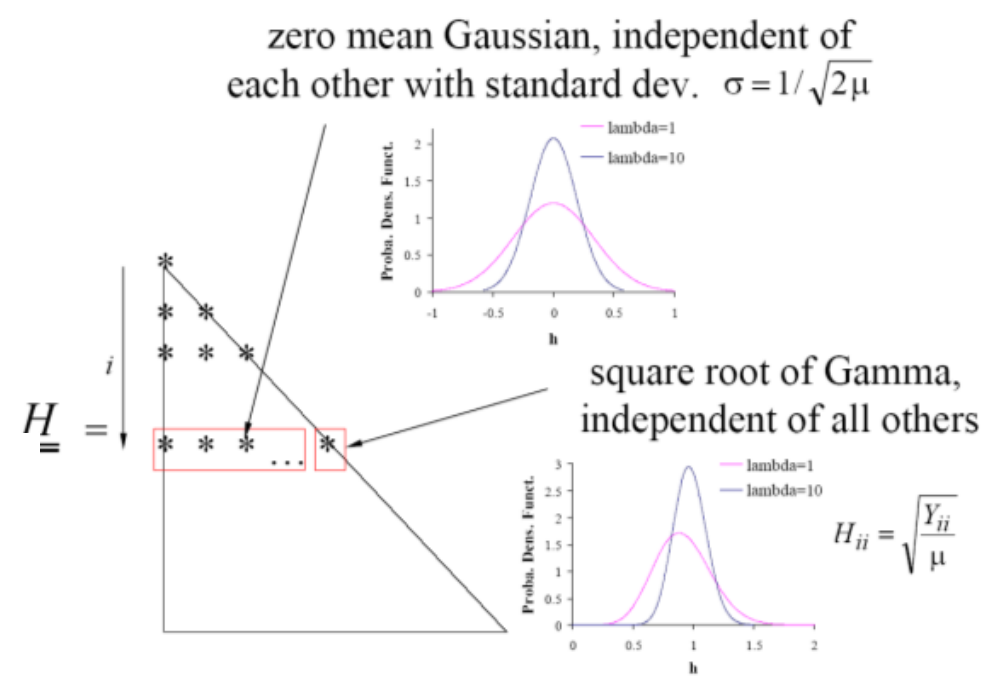

Figure 1. Structure of the random $\boldsymbol{H}$ matrices with $n=8, i=2$, and $\lambda_{0}=1$ and 10.

(1) all of its non-zero elements $H_{i l}$ are independent random variables,

(2) its off-diagonal elements $H_{i l}, i \neq l$, are normally distributed (Gaussian) random variables with standard deviation $\sigma=1 / \sqrt{2 \mu}$, and

(3) its diagonal elements $H_{i i}$ are obtained as $H_{i i}=\sqrt{Y_{i i} / \mu}$ where $Y_{i i}$ is Gamma distributed with parameter $(p(i)-1) / 2$ where

$$
p(i)=n-i+2 \lambda_{0}-1 \text { and } \mu=\left(n+2 \lambda_{0}-1\right) / 2
$$

In the above equations, $n$ is the size of the matrices and the parameter $\lambda_{0}>0$ is the free parameter of the statistical distribution of the random matrices $A$. An alternative parametrization is through the dispersion parameter $\delta$ defined as

$$
\delta^{2}=\frac{n+1}{n+2 \lambda_{0}-1} .
$$


As stated above, a key component of the maximum entropy formulation is the satisfaction of the physical constraints that the ROM parameters must satisfy. While this issue is well understood for the stiffness matrix of linear structural dynamics, it is not as obvious for the combination of linear, quadratic, and cubic stiffness coefficients of the ROM of Eq. (4). The derivation of such a condition was achieved in [10] based on integral expressions off the stiffness coefficients of Eq. (4) obtained as a by-product of the derivation of this governing equation. Specifically, it was found that

$$
\begin{aligned}
K_{m n}^{(1)} & =\int_{\Omega_{0}} \frac{\partial U_{i}^{(m)}}{\partial X_{k}} C_{i k l p} \frac{\partial U_{l}^{(n)}}{\partial X_{p}} d \boldsymbol{X} \\
K_{m n p}^{(2)} & =\frac{1}{2}\left[\hat{K}_{m n p}^{(2)}+\hat{K}_{p m n}^{(2)}+\hat{K}_{n p m}^{(2)}\right]
\end{aligned}
$$

where

$$
\hat{K}_{m n p}^{(2)}=\int_{\Omega_{0}} \frac{\partial U_{i}^{(m)}}{\partial X_{j}} C_{i j k l} \frac{\partial U_{r}^{(n)}}{\partial X_{k}} \frac{\partial U_{r}^{(p)}}{\partial X_{l}} d \boldsymbol{X}
$$

and

$$
K_{\text {msnp }}^{(3)}=\frac{1}{2} \int_{\Omega_{0}} \frac{\partial U_{i}^{(m)}}{\partial X_{j}} \frac{\partial U_{i}^{(s)}}{\partial X_{k}} C_{j k l w} \frac{\partial U_{r}^{(n)}}{\partial X_{l}} \frac{\partial U_{r}^{(p)}}{\partial X_{w}} d X .
$$

In these equations, $\Omega_{0}$ denotes the domain of the structure in the undeformed configuration, $X \in \Omega_{0}$. Next, a reshaping was first performed to transforms the $M \times M x M$ third order tensor $\hat{\boldsymbol{K}}^{(2)}$ into a $M \times M^{2}$ rectangular array $\tilde{\boldsymbol{K}}^{(2)}$ and the $M \times M x M x M$ fourth order tensor $\boldsymbol{K}^{(3)}$ into a $M^{2} \mathrm{x}^{2}$ square matrix $\tilde{\boldsymbol{K}}^{(\mathbf{3})}$. These operations are achieved as follows:

$$
\tilde{K}_{m J}^{(2)}=\hat{K}_{m n p}^{(2)} \quad \text { with } \quad J=(n-1) M+p
$$

and

$$
\tilde{K}_{I J}^{(3)}=K_{m s n p}^{(3)} \quad \text { with } \quad I=(m-1) M+s \text { and } J=(n-1) M+p .
$$

With these operations, it was shown that the matrix $\overline{\boldsymbol{K}}_{B}$ defined as

$$
\overline{\boldsymbol{K}}_{B}=\left[\begin{array}{cc}
\boldsymbol{K}^{(1)} & \tilde{\boldsymbol{K}}^{(2)} \\
\tilde{\boldsymbol{K}}^{(2) T} & 2 \tilde{\boldsymbol{K}}^{(3)}
\end{array}\right]
$$

is positive definite.

Having established the above property, it seems that the modeling of uncertainty of the ROM level for nonlinear geometric structure is now well defined. Specifically [10],

(1) from a finite element model of the structure, identify the parameters $K_{i j}^{(1)}, \bar{K}_{i j l}^{(2)}$ and $\bar{K}_{i j l p}^{(3)}$

(2) determine the coefficients $K_{i j l}^{(2)}$ and $K_{i j l p}^{(3)}$ by “inverting” the Eqs (6a) and (6b) with Eq. (7)

(3) determine the coefficients $\hat{K}_{m n p}^{(2)}$ from Eq. (19)

(4) form the matrices $\widetilde{\boldsymbol{K}}^{\mathbf{( 2 )}}$ and $\widetilde{\boldsymbol{K}}^{\mathbf{( 3 )}}$ from Eqs (22) and (23), then $\overline{\boldsymbol{K}}_{B}$ from Eq. (24)

(5) set $\overline{\boldsymbol{A}}=\overline{\boldsymbol{K}}_{B}$ and follow Eqs (14)-(16) and Fig. 1 to generate random samples of the matrix $\boldsymbol{A}=\boldsymbol{K}_{B}$ 
(6) proceed with steps (4), (3), (2) and (1) in reverse with each sample of $\boldsymbol{K}_{B}$ to obtain realizations of the uncertain linear, quadratic, and cubic stiffnesses $K_{i j}^{(1)}, \bar{K}_{i j l}^{(2)}$ and $\bar{K}_{i j l p}^{(3)}$ denoted as $\breve{K}_{i j}^{(1)}$, $\breve{K}_{i j l}^{(2)}$ and $\breve{K}_{i j l p}^{(3)}$ from which the sample of the uncertain response can be determined.

\section{THE CHALLENGES}

The process defined by steps (1)-(6) above seems well defined but after a closer inspection and trials, two key challenges were encountered:

(I) the determination of the parameters $\hat{K}_{m n p}^{(2)}$ and $K_{i j l p}^{(3)}$ cannot be uniquely performed from Eqs (6a), (6b), (7), and (19) as there are more unknowns than equations.

(II) when the above determination can be carried out, the resulting mean model matrix $\overline{\boldsymbol{K}}_{B}$ may not be positive definite when the ROM parameters $K_{i j}^{(1)}, \bar{K}_{i j l}^{(2)}$ and $\bar{K}_{i j l p}^{(3)}$ are identified from some finite element codes, e.g., from Nastran.

The positive definiteness of $\overline{\boldsymbol{K}}_{B}$ stems, see [10], from the positive definiteness of the potential energy in the structure, it is thus an essential property and its violation may lead to unphysical behavior.

An example of this situation has been encountered with a flat cantilevered beam, see [11] and details below, for which a 2-basis function model was derived. The first of these basis function was selected as the first linear mode of the beam and the second as its associated dual (see definition/discussion in [3,5,12]) which exhibits only inplane motions. Performing the identification of this model nonintrusively from a Nastran finite element model using the approaches of $[4,5]$ led to stiffness parameters that were very robust with respect to the identification details. Among these parameters, it was found that $\bar{K}_{1122}^{(3)}$ was rather large and negative [12]. However, from Eqs (6b) and (7) this parameter should equal $K_{1122}^{(3)}+2 K_{1212}^{(3)}$ where $K_{1212}^{(3)}$ must be positive (it is a diagonal term of $\overline{\boldsymbol{K}}_{B}$ ) and $K_{1122}^{(3)}$ should be positive according to Eq. (21). Interestingly, the parameter $\bar{K}_{1122}^{(3)}$ identified using the finite element formulation of [13] is positive.

This example clearly demonstrates that there may be an inconsistency between the expressions of Eqs (6),(7),(18)-(21) and the ROM parameters identified from the finite element software. They may affect the positive definiteness of $\overline{\boldsymbol{K}}_{B}$ but can also have significant implications regarding the accuracy of the ROM. For example, shown in Fig. 2 are the modal force along mode 1 vs. tip displacement predictions from Nastran and two identified ROMs for the cantilevered beam subjected to a uniform transverse load along its span. The Nastran nonlinear static predictions (from SOL 106) show an almost perfectly linear relation. However, the ROM identified from this software, with the negative $\bar{K}_{1122}^{(3)}$ exhibits an unphysical behavior: there exists a peak of the force at a certain displacement level and thus, for sufficiently large load down, the tip of the beam is predicted to move up! This situation does not happen with the nonlinear finite element code Bobtran implementing the formulation of [13] and for which $\bar{K}_{1122}^{(3)}$ is slightly positive. Those prediction lead to a slight stiffening of the beam in the load range considered. 


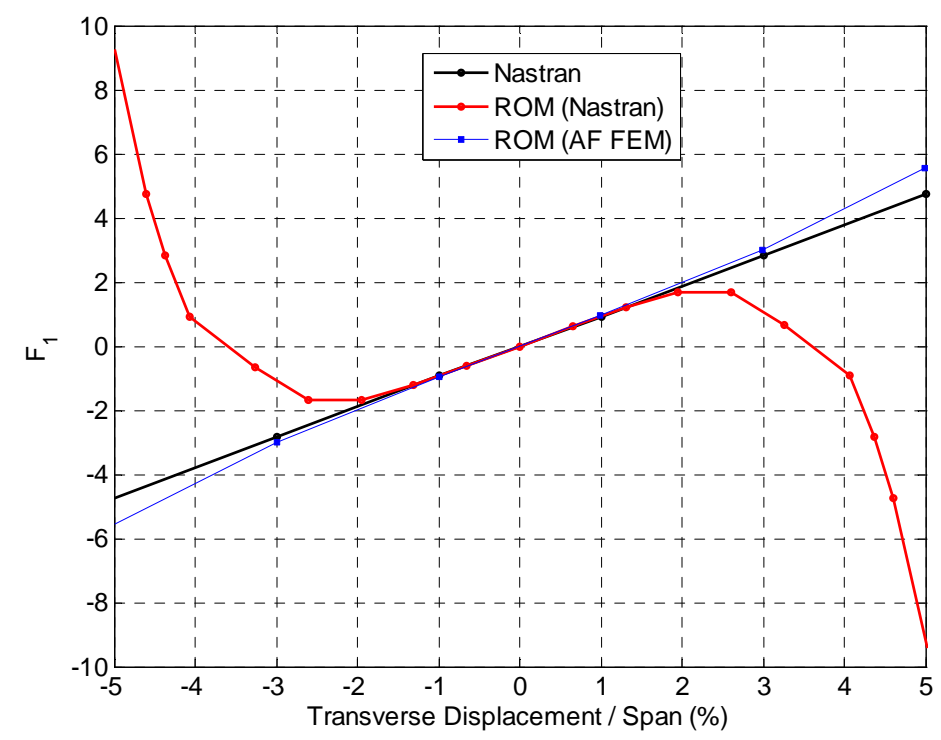

Figure 2. Modal force along mode 1 vs. tip transverse displacement for a flat cantilevered beam. Results from Nastran nonlinear static (SOL 106), the ROM identified from the Nastran model ("ROM(Nastran)”) and from the Air Force code Bobtran (“ROM(AF FEM)”) implementing [13].

\section{RESOLUTION OF THE CHALLENGES}

\subsection{Overall Perspective}

The two challenges (I) and (II) above were addressed jointly as follows.

(a) Stiffness parameters for which the "inversion" of Eqs (6a), (6b), (7), and (19) could not be accomplished uniquely were optimized to render the matrix $\overline{\boldsymbol{K}}_{B}$ as positive definite as possible.

(b) If the resulting matrix $\overline{\boldsymbol{K}}_{B}$ is not positive definite, its "least important" elements were modified as little as possible to make it positive definite. After the matrix $\overline{\boldsymbol{K}}_{B}$ has been modified, the modified mean model predictions were determined and compared with the ones resulting from the originally identified parameters. The modified matrix $\overline{\boldsymbol{K}}_{B}$ was accepted if the difference in predictions was found small enough. The introduction of uncertainty then followed as in Eqs (14)-(16) and Fig. 1.

(c) If the predictions obtained from the modified mean model differed too significantly from those obtained with the originally identified parameters, a modified simulation approach was defined that relies on the $\overline{\boldsymbol{K}}_{B}$ matrix resulting from step (a) above.

\subsection{Optimized Decomposition}

The first step, (a), of the above process focused on the extraction of the parameters $\hat{K}_{i j l}^{(2)}$ and $K_{i j l p}^{(3)}$ from the identified ones $\bar{K}_{i j l}^{(2)}$ and $\bar{K}_{i j l p}^{(3)}$ as to render the matrix $\overline{\boldsymbol{K}}_{B}$ as positive definite as possible.

Consider first the parameters for which all indices are equal, i.e., $\hat{K}_{i i i}^{(2)}$ and $K_{i i i i}^{(3)}$. For these, no indeterminacy exists and

$$
\hat{K}_{\text {iii }}^{(2)}=\frac{2}{3} \bar{K}_{\text {iii }}^{(2)} \quad \text { and } \quad K_{\text {iiii }}^{(3)}=\bar{K}_{\text {iiii }}^{(3)} .
$$

For coefficients depending on two mode indices $i$ and $j>i$, the indeterminacy exists as there are 4 new quadratic coefficients, $\hat{K}_{i j j}^{(2)}, \hat{K}_{i i j}^{(2)}, \hat{K}_{j i j}^{(2)}, \hat{K}_{j i i}^{(2)}$, and 4 new cubic ones, $K_{i i i j}^{(3)}, K_{i i j j}^{(3)}, K_{i j i j}^{(3)}, K_{i j j j}^{(3)}$, 
considering the symmetry of Eq. (7) as well as the property $\hat{K}_{i j l}^{(2)}=\hat{K}_{i l j}^{(2)}$ resulting from Eq. (19). There are however only 5 independent equations relating them:

$$
\begin{gathered}
\bar{K}_{i i j}^{(2)}=2 \hat{K}_{i i j}^{(2)}+\hat{K}_{j i i}^{(2)} \quad \text { and } \quad \bar{K}_{i j j}^{(2)}=\frac{1}{2} \hat{K}_{i j j}^{(2)}+\hat{K}_{j i j}^{(2)} \\
\bar{K}_{i i i j}^{(3)}=3 K_{i i i j}^{(3)} \quad \bar{K}_{i i j j}^{(3)}=K_{i i j j}^{(3)}+2 K_{i j i j}^{(3)} \quad \text { and } \quad \bar{K}_{i j j j}^{(3)}=K_{i j j j}^{(3)} .
\end{gathered}
$$

To resolve the indeterminacy, it is desired that the matrix $\overline{\boldsymbol{K}}_{B}$ be made as positive definite as possible given the constraints of Eqs (26). Since the split of say $\bar{K}_{i i j}^{(2)}$ into $\hat{K}_{i i j}^{(2)}$ and $\hat{K}_{j i i}^{(2)}$ should not be dependent on the coefficients of other modes than $i$ and $j$, it is more specifically imposed that the unknown coefficients maximize the lowest eigenvalue of $\overline{\boldsymbol{K}}_{B, i j}^{(2)}$ which is the $6 \times 6 \overline{\boldsymbol{K}}_{B}$ matrix built using the coefficients relevant to modes $i$ and $j$ only. This optimization is performed for all distinct combinations of $i$ and $j>i$ modes which then leads to all parameters $\hat{K}_{i j l}^{(2)}$ and $K_{i j l p}^{(3)}$ with 2 different indices.

For the coefficients involving 3 different indices, $i, j>i$ and $k>j$, a similar effort is carried out: 3 new quadratic coefficients related by 1 independent equation, and 2 new cubic ones related by 1 independent equation and the indeterminacy is resolved by maximizing the lowest eigenvalue of $\overline{\boldsymbol{K}}_{B, i j k}^{(3)}$ which is the $12 \times 12 \overline{\boldsymbol{K}}_{B}$ matrix built using the coefficients relevant to modes $i$, $j$, and $k$ only. This effort is then repeated for all distinct combinations of $k>j>i$ modes which then leads to all parameters $\hat{K}_{i j l}^{(2)}$ and $K_{i j l p}^{(3)}$ with 3 different indices.

It remains to address the determination of the parameters $K_{i j l p}^{(3)}$ with all 4 indices different. For each set of the 4 indices, there are only 3 such distinct parameters taking into account Eq. (7), i.e., $K_{i j l p}^{(3)}$, $K_{i l j p}^{(3)}$, $K_{i p j l}^{(3)}$ assuming $p>l>j>i$ but only 1 independent equation

$$
\bar{K}_{i j l p}^{(3)}=2 K_{i j l p}^{(3)}+2 K_{i l j p}^{(3)}+2 K_{i p j l}^{(3)} .
$$

As before, the indeterminacy is resolved by maximizing the lowest eigenvalue of $\overline{\boldsymbol{K}}_{B, i j l p}^{(4)}$ which is the $20 \times 20 \overline{\boldsymbol{K}}_{B}$ matrix built using the coefficients relevant to modes $i, j, l$ and $p$ only. This effort is then repeated for all distinct combinations of $p>l>j>i$ modes to yield the remaining cubic parameters $K_{i j l p}^{(3)}$.

\subsection{Rendering $\overline{\boldsymbol{K}}_{B}$ positive definite}

After the series of optimization efforts carried out in the previous section, the resulting matrix $\overline{\boldsymbol{K}}_{B}$ may be positive definite in which case the uncertainty modeling can proceed as in Eqs (14)-(16) and Fig. 1. If this matrix is not positive definite, it will be modified in this second step to become positive definite. This modification will be accomplished:

(a) without affecting the part of $\overline{\boldsymbol{K}}_{B}$ that is positive definite, e.g., the linear stiffness matrix $\boldsymbol{K}^{(1)}$, and

(b) inducing the smallest changes possible to this matrix. 
The task (a) has been achieved iteratively by constructing the biggest block of the original matrix $\overline{\boldsymbol{K}}_{B}$ that is positive definite. This block is at least of size $N$ since the linear stiffness matrix $\boldsymbol{K}^{(1)}$ is positive definite. Accordingly, the top left block of $\overline{\boldsymbol{K}}_{B}$ of size $N+1$ is first considered and it is checked for positive definiteness (e.g., by constructing its Cholesky decomposition). If it is positive definite, the algorithm moves to the top left block of size $N+2$ and the process is repeated.

Otherwise, a permutation of the rows and columns $N+1$ and $N+2$ is performed. If the top left block of size $N+1$ is now positive definite, the algorithm accepts the permutation and moves forward to the top left block of size $N+2$. On the contrary, the permutation between rows $N+1$ and $N+2$ is reversed and a permutation of rows $N+1$ and $N+3$ is performed followed by a positive definiteness check. This process concludes when no permutation of rows and columns achieves an increase in the size of the top left block of $\overline{\boldsymbol{K}}_{B}$ which is positive definite.

At that point, the matrix $\overline{\boldsymbol{K}}_{B}$ has been transformed in a symmetric matrix $\tilde{\boldsymbol{K}}_{B}$ which has the form

$$
\tilde{\boldsymbol{K}}_{B}=\left[\begin{array}{ll}
\boldsymbol{K}_{11} & \boldsymbol{K}_{12} \\
\boldsymbol{K}_{12}^{T} & \boldsymbol{K}_{22}
\end{array}\right]
$$

where $\boldsymbol{K}_{11}$ is positive definite and of size $N_{p}, \boldsymbol{K}_{12}$ is of size $N_{p} \times N_{r}$, and $\boldsymbol{K}_{22}$ is of size $N_{r} \times N_{r}$ where $N_{r}=N^{2}+N-N_{p}$.

The task (b) above then proceeds with replacing the matrix $\tilde{\boldsymbol{K}}_{B}$ by

$$
\hat{\boldsymbol{K}}_{B}=\left[\begin{array}{ll}
\boldsymbol{K}_{11} & \boldsymbol{K}_{12} \\
\boldsymbol{K}_{12}^{T} & \boldsymbol{K}_{22}
\end{array}\right]+\left[\begin{array}{cc}
0 & \Delta_{1} \\
\Delta_{1}^{T} & \Delta_{2}
\end{array}\right]=\tilde{\boldsymbol{K}}_{B}+\Delta
$$

where the matrix $\Delta$ will be selected to have the minimum Frobenius norm under the constraint that $\hat{\boldsymbol{K}}_{B}$ is at least positive semidefinite. The solution of this nonlinear optimization problem will be obtained iteratively through a sequence of linear optimization problems in which the positive definiteness constraint is enforced linearly. To this end, note that a symmetric perturbation $\delta \boldsymbol{A}$ of a symmetric matrix $\boldsymbol{A}$ leads to a first order perturbation of any of its non repeated eigenvalues $\lambda$ by [14]

$$
\delta \lambda=\psi^{T} \delta A \psi
$$

where $\psi$ is the normalized, $\psi^{T} \psi=1$, eigenvector of $\boldsymbol{A}$ corresponding to the eigenvalue $\lambda$. Then, assuming that $\Delta$ is small enough for Eq. (30) to apply, the eigenvalues $\hat{\lambda}_{i}$ of $\hat{\boldsymbol{K}}_{B}$ can be expressed as

$$
\hat{\lambda}_{i}=\tilde{\lambda}_{i}+\tilde{\psi}_{i}^{T} \Delta \tilde{\Psi}_{i}
$$

where $\tilde{\lambda}_{i}$ and $\tilde{\psi}_{i}$ are the eigenvalues and corresponding normalized eigenvectors of $\tilde{\boldsymbol{K}}_{B}$. Then, the positive semidefinite requirement can be approximately written as

$$
\tilde{\Psi}_{i}^{T} \Delta \tilde{\Psi}_{i} \geq-\tilde{\lambda}_{i} \text { for all } i \text { such that } \tilde{\lambda}_{i} \leq 0 \text {. }
$$

Adopting these linearized constraints, the determination of $\Delta$ can be rewritten as the minimization of

$$
\|\Delta\|_{F}^{2}-2 \sum_{\tilde{\lambda}_{i}<0} \mu_{i}\left[\tilde{\Psi}_{i}^{T} \Delta \tilde{\Psi}_{i}+\tilde{\lambda}_{i}\right]
$$


where $\|\cdot\|_{F}$ denotes the Frobenius norm and $\mu_{i}$ are the Lagrange multiplier associated to the equality constraints of Eq. (32).

Differentiating the objective function of Eq. (33) yields the linear system of equations

$$
\Delta_{1}=\sum_{\tilde{\lambda}_{i}<0} \mu_{i} \tilde{\Psi}_{i}^{(1)}\left[\tilde{\Psi}_{i}^{(2)}\right]^{T}
$$

and

$$
\Delta_{2}=\sum_{\tilde{\lambda}_{i}<0} \mu_{i} \tilde{\Psi}_{i}^{(2)}\left[\tilde{\Psi}_{i}^{(2)}\right]^{T}
$$

where the eigenvectors $\tilde{\Psi}_{i}$ are partitioned into vectors $\tilde{\Psi}_{i}^{(1)}$ and $\tilde{\Psi}_{i}^{(2)}$ of $N_{p}$ and $N_{r}$ components, respectively. That is, $\tilde{\Psi}_{i}^{T}=\left[\left[\tilde{\Psi}_{i}^{(1)}\right]^{T}\left[\tilde{\Psi}_{i}^{(2)}\right]^{T}\right]$. Finally, the constraints of Eq. (32) yield

$$
\sum_{\tilde{\lambda}_{r}<0}\left(2 a_{r i} b_{r i}+b_{r i}^{2}\right) \mu_{r}=-\tilde{\lambda}_{i} \text { with } a_{r s}=\left[\tilde{\Psi}_{r}^{(1)}\right]^{T} \tilde{\Psi}_{s}^{(1)} \text { and } b_{r s}=\left[\tilde{\Psi}_{r}^{(2)}\right]^{T} \tilde{\psi}_{s}^{(2)}
$$

Solving the linear system of equations (36) yields the values of the Lagrange multipliers $\mu_{i}$ which can then be reintroduced in Eq. (34) and (35) to yield the unknown partitions $\Delta_{1}$ and $\Delta_{2}$ of $\Delta$.

The resulting matrix $\hat{\boldsymbol{K}}_{B}$ will then in general not be positive definite but the process can be repeated with a new $\tilde{\boldsymbol{K}}_{B}=\hat{\boldsymbol{K}}_{B}$ until a matrix $\hat{\boldsymbol{K}}_{B}$ positive definite/semidefinite is finally obtained. At that point, the rows/columns permutations performed to obtain the largest block positive definite are reversed leading to a matrix $\boldsymbol{K}_{B}^{\prime}$ which is positive definite and thus could serve as the basis for the uncertainty modeling according to Eqs (14)-(16) and Fig. 1.

It remains however to assess if rendering the matrix positive definite has changed "significantly" the mean model to the point that the uncertainty analysis may not be relevant to the original mean model. To this end, the quadratic and cubic stiffness coefficients $\hat{K}_{i j l}^{\prime(2)}$ and $K_{i j l p}^{\prime(3)}$ are extracted from $\boldsymbol{K}_{B}^{\prime}$ and used, with the linear coefficients $K_{i j}^{(1)}$, which have not been modified by the modification of the matrix $\tilde{\boldsymbol{K}}_{B}$, to compute a set of representative responses of this modified mean model. These responses can then be compared with those from the identified model to assess whether the matrix $\boldsymbol{K}_{B}^{\prime}$ is appropriate to carry out the uncertainty analysis.

\section{$5.4 \overline{\boldsymbol{K}}_{B}$ cannot be made positive definite without affecting the model}

For most of the structural models investigated so far, see the application section for details, the procedure described in the previous section yielded a matrix $\boldsymbol{K}_{B}^{\prime}$ that closely represents the originally identified mean model and thus can be used to carry out the uncertainty analysis. When this is not the case, however, it is proposed here to modify the modeling approach of Eqs (14) and (15) using a $L D L^{T}$ decomposition [15] in place of the Cholesky one so that it can be applied to the matrix $\overline{\boldsymbol{K}}_{B}$ resulting from the optimized decomposition. Specifically, this matrix is first rewritten as

then, the uncertain matrices are obtained as

$$
\overline{\boldsymbol{K}}_{B}=\overline{\boldsymbol{L}}_{\boldsymbol{K}} \boldsymbol{D} \overline{\boldsymbol{L}}_{\boldsymbol{K}}^{\boldsymbol{T}}
$$




$$
\boldsymbol{K}_{B}=\overline{\boldsymbol{L}}_{\boldsymbol{K}} \boldsymbol{H} \boldsymbol{D} \boldsymbol{H}^{T} \overline{\boldsymbol{L}}_{\boldsymbol{K}}^{T}
$$

where $\boldsymbol{H}$ is the same matrix as in Eq. (15) and Fig. 1. Note in Eq. (37) that the diagonal matrix $\boldsymbol{D}$ will be selected to only carry a sign, i.e., its elements are either +1 or -1 only.

\subsection{Uncertainty modeling of the largest positive block only}

An alternative to the modifications of sections 5.3 and 5.4 is to proceed with the modeling of uncertainty only on the largest part of the model that is consistent with the theory of Eqs (18)-(24) leaving the rest of it equal to the mean model. That is, uncertainty is introduced on the positive definite block $\boldsymbol{K}_{11}$ of Eq. (28) while leaving the corresponding matrices $\boldsymbol{K}_{12}$ and $\boldsymbol{K}_{22}$ unchanged, equal to their values resulting for example from the optimization of Eqs (29)-(36) or as the initial conditions of this process. When the eigenvalues of the matrices $\tilde{\boldsymbol{K}}_{B}$ and $\hat{\boldsymbol{K}}_{B}$ differ from those of $\boldsymbol{K}_{11}$ only mostly by the addition of small, positive or negative, eigenvalues, this process could be viewed as similar to the approach of [9] in focusing the uncertainty on the dominant component of the model only.

\section{APPLICATIONS}

The above developments were applied to a series of structures for which mean NLROM were obtained in prior investigations. While all of these applications focus on static responses, uncertain dynamic responses could be computed with the same set of uncertain stiffness coefficients as those used for the static problems.

The first example considered is the cantilevered straight beam discussed earlier, see Fig. 2, in the context of the positive definiteness of $\overline{\boldsymbol{K}}_{B}$ and the existence of inconsistencies between the stiffness coefficients obtained from commercial finite element software (Nastran here) and Eqs (18)-(21). The mean model selected here includes 8 basis functions - the first 4 linear modes and 4 duals as constructed in [16]. The matrix $\overline{\boldsymbol{K}}_{B}$ obtained after the optimized decomposition was not positive definite. The application of the procedure of Eqs (28)-(36) did render it positive definite and the predictions obtained from that modified mean model were found to be very close to those from the originally identified model. The uncertainty analysis was then performed using Eqs (14) and (15) with an overall uncertainty level selected to be $\delta=0.30$ and shown in Fig. 4 are the transverse and inplane displacements at the tip vs. load level for the mean model as well as 5th-95th percentile uncertainty band.

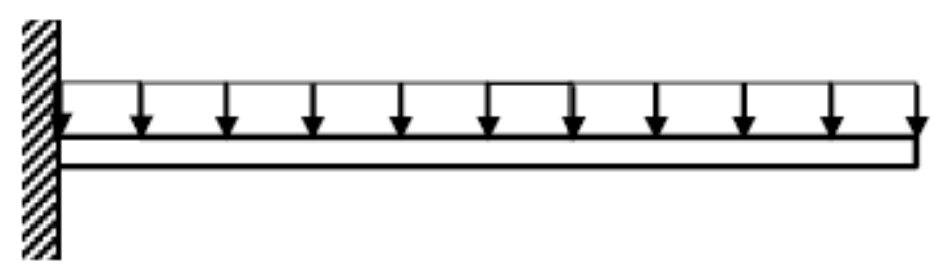

Figure 3. Cantilevered straight beam with uniform loading. 

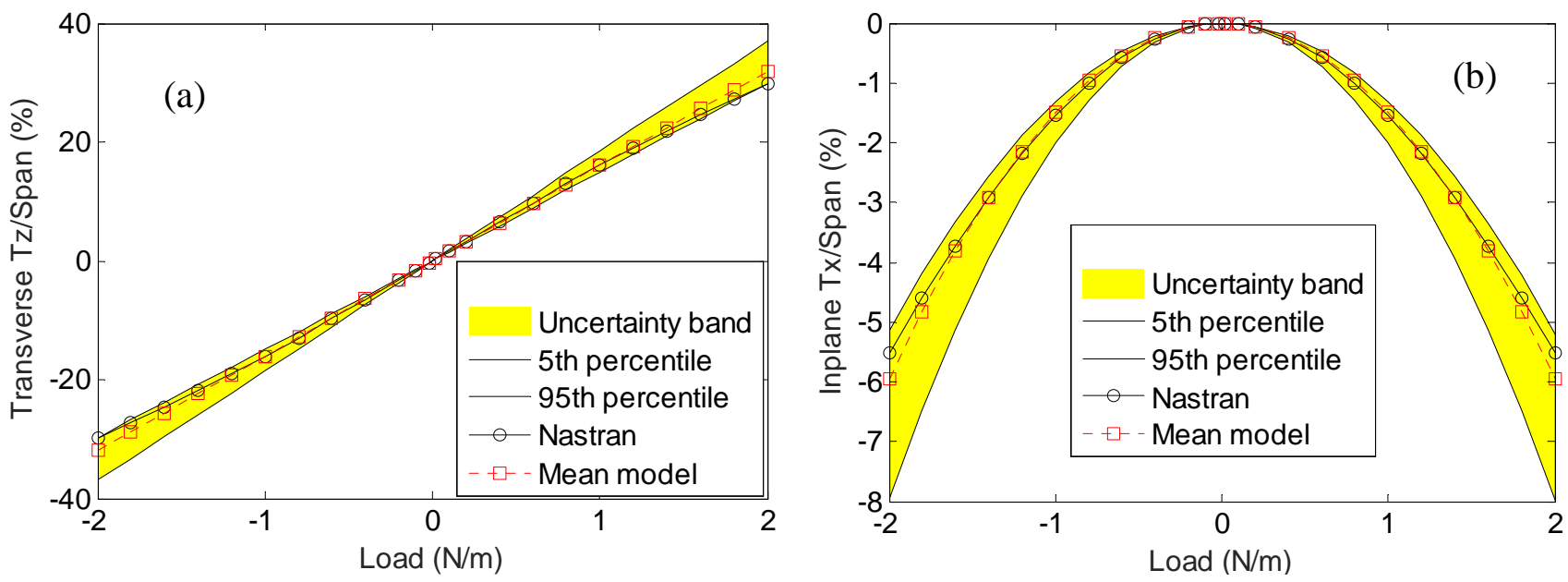

Figure 4. Static displacement of the cantilevered straight beam tip under a uniform loading. (a) Transverse and (b) inplane displacements (percent of span) vs. load. Mean and uncertain models.

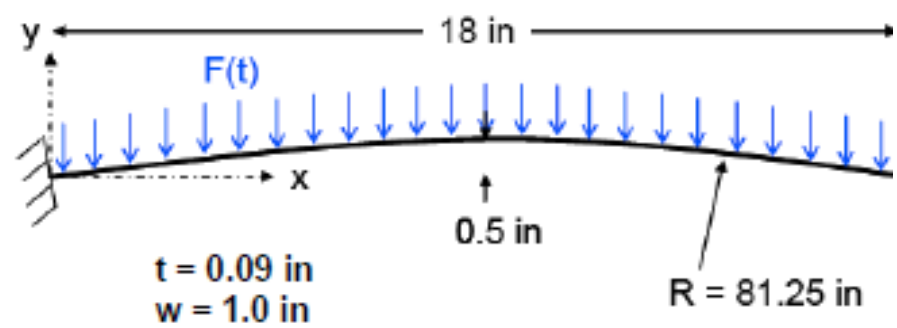

Figure 5. Cantilevered curved beam with uniform loading.
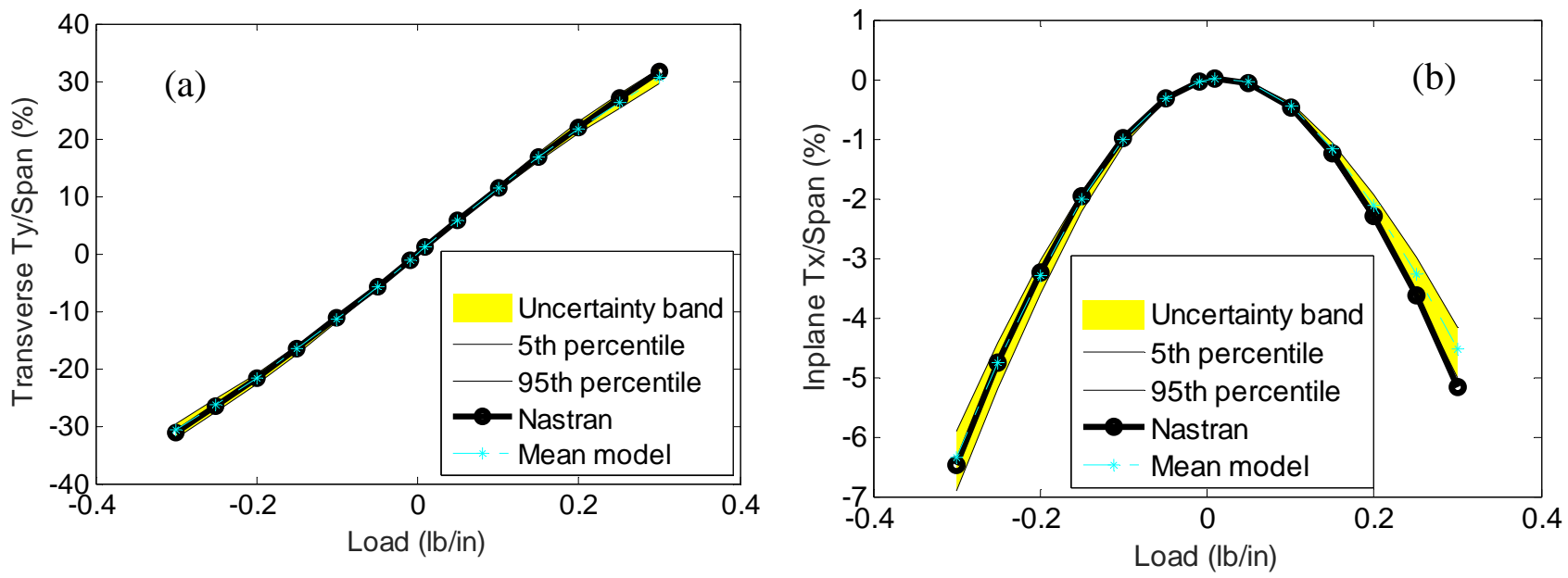

Figure 6. Static displacement of the cantilevered curved beam tip under uniform loading. (a) Transverse and (b) inplane displacements (percent of span) vs. load. Mean and uncertain models.

The process was next repeated with the curved cantilevered beam of Fig. 5 the response of which was modeled using 9 basis functions - the first 3 linear modes and 6 duals. As for the straight cantilevered beam, the matrix $\overline{\boldsymbol{K}}_{B}$ obtained after the optimized decomposition was not positive definite. Again, the application of the procedure of Eqs (28)-(36) did render it positive definite and the predictions obtained from that modified mean model were found to be very close to those from the originally identified model. The uncertainty analysis was then performed using Eqs (14) and (15) with an overall uncertainty level selected to be $\delta=0.15$ and shown in Fig. 6 are the transverse (vertical) and inplane 
(horizontal) displacements at the tip vs. load level for the mean model as well as 5th-95th percentile uncertainty band. Comparing these results with those of Fig. 4, it is concluded that the response of the curved beam is much less sensitive to uncertainty than its straight counterpart.
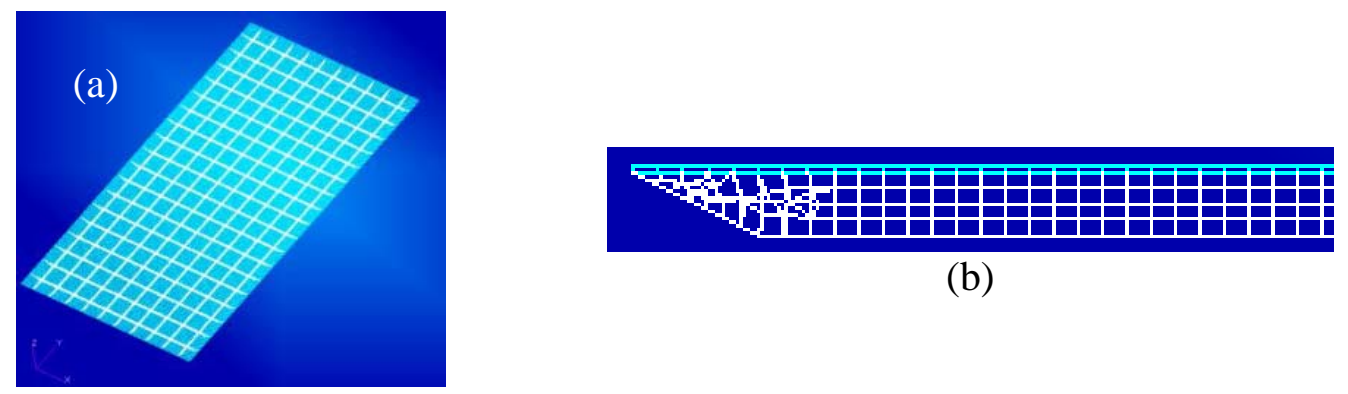

(b)

Figure 7. Orthogrid panel considered in [17] (a) Perspective view and (b) cross-section.
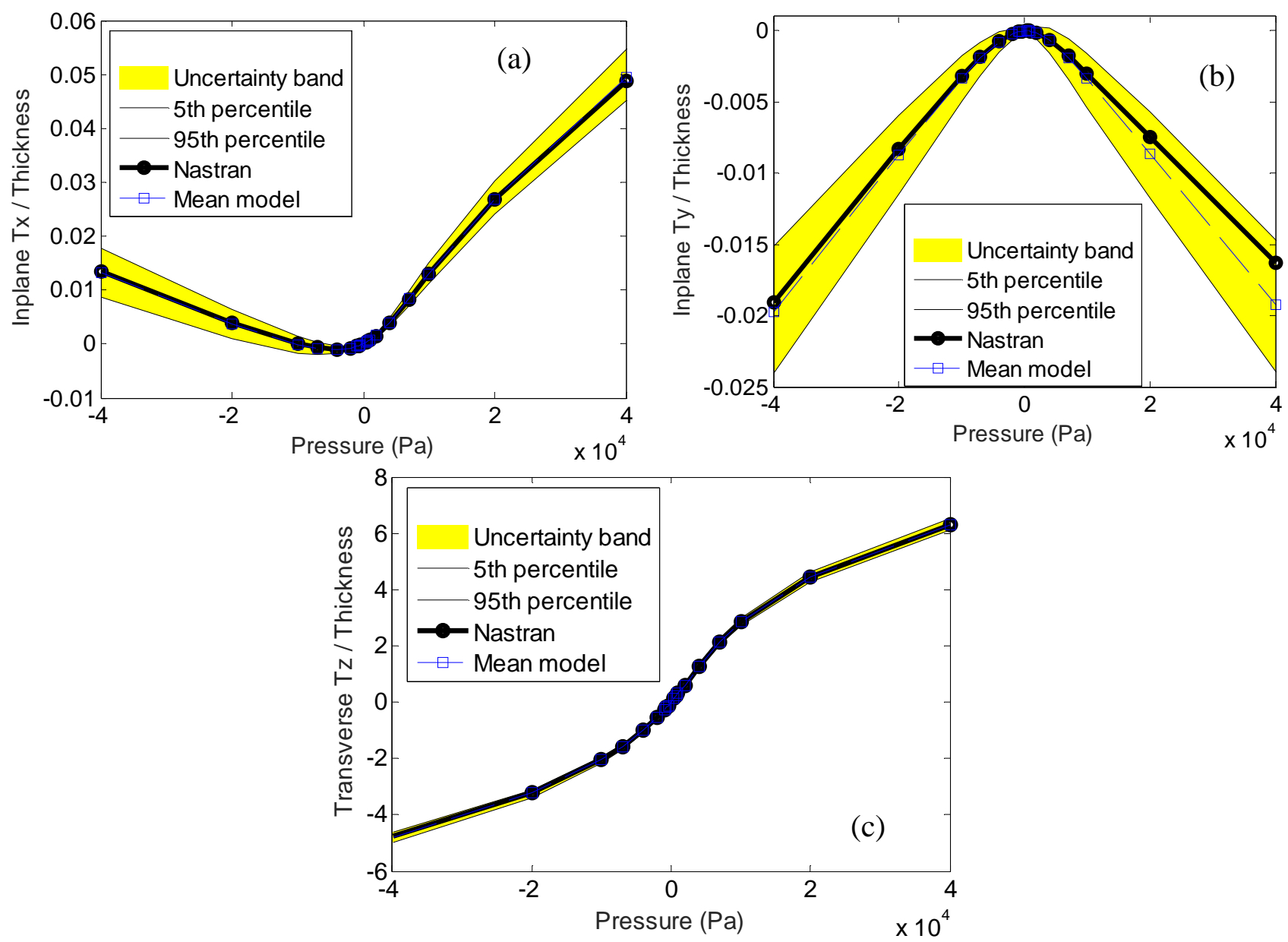

Figure 8. Static displacement of the orthogrid panel under a uniform loading. (a) Inplane $x$, (b) inplane $y$, and (c) transverse $z$ displacements at a quarter point divided by the panel thickness vs. load. Mean and uncertain models.

The above process was also repeated for the orthogrid panel of Fig. 7 [17] modeled using 17 basis functions - the first 8 linear modes and 9 duals. The uncertainty analysis was carried out as for the two previous examples with a value $\delta=0.31$. Then, shown in Fig. 8 are the displacements at a quarter point 
along the three directions for the mean and uncertain models. It is interesting to observe that the inplane displacements are much more sensitive to the uncertainty than the transverse ones.

The application of the above concepts was also performed on the clamped-clamped curved beam of Fig. 9 in a range of displacements that includes its snap-through. Proceeding as before, the matrix $\overline{\boldsymbol{K}}_{B}$ obtained after the optimized decomposition was not positive definite but the application of the procedure of Eqs (28)-(36) did render it positive definite. However, the predictions obtained from that modified mean model were found to be too different from those from the originally identified model, in particular that modified model did not snap through as predicted by either the original NLROM or the finite element model. In this light, the revised uncertainty modeling of Eqs. (37) and (38) was applied with $\delta=0.026$. Then, shown in Fig. 10 are the transverse (vertical) and inplane (horizontal) displacements at a beam quarter point vs. load level for the mean model as well as 5th-95th percentile uncertainty band. As already observed in connection with the two previous examples, the inplane response of the curved beam appears more sensitive to uncertainty than its transverse counterpart. Note as well the large increase in the uncertainty band post snap through, in both directions, with much smaller variations of the snap through load which are nevertheless much larger than the variability of the response prior to snap through.

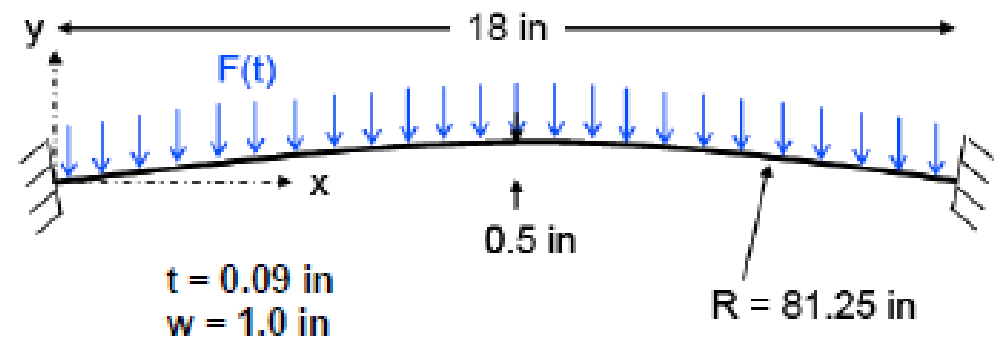

Figure 9. Clamped-clamped curved beam with uniform loading.
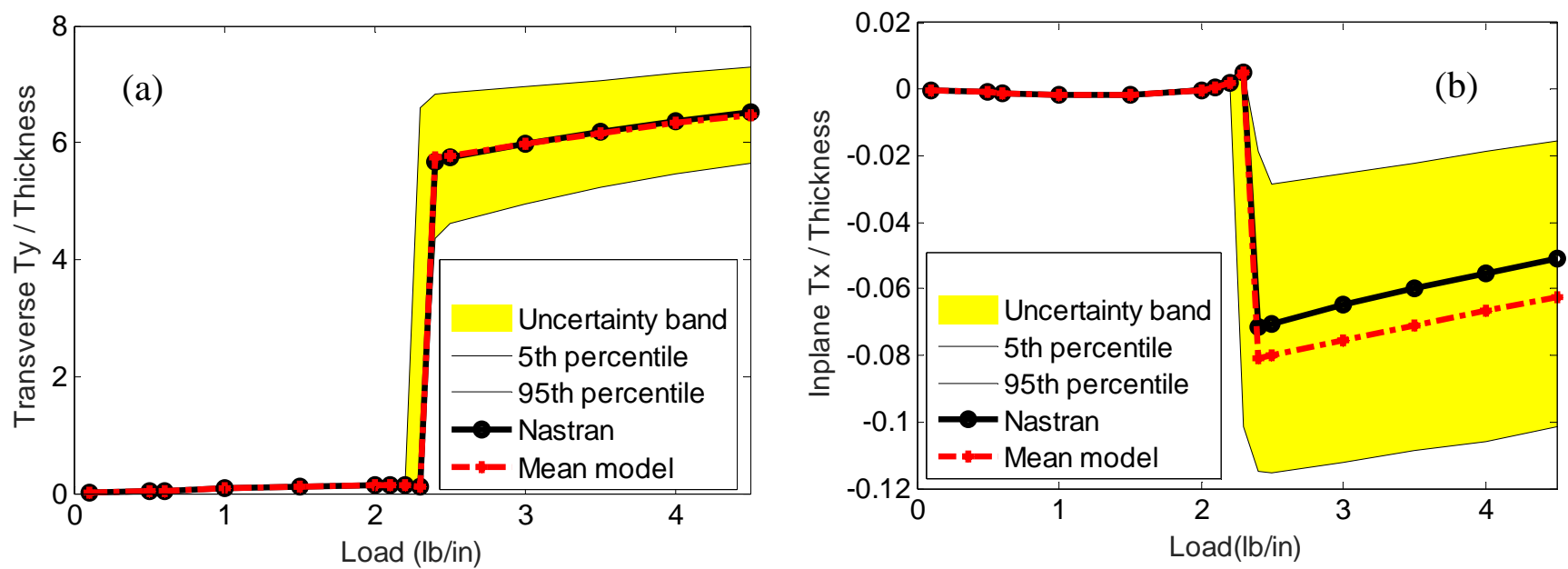

Figure 10. Static displacement of the clamped-clamped curved beam under a uniform loading. (a) Transverse and (b) inplane displacements (in percent of span) of a beam quarter point vs. load. Mean and uncertain models.

The above results were all obtained using the modifications of section 5.3 and 5.4 but an assessment of the approach of section 5.5, i.e., the uncertainty modeling of the matrix $\boldsymbol{K}_{11}$ of Eq. (28), was also performed for the clamped-clamped curved beam of Fig. 9, see Fig. 11 with the same value of $\delta=0.026$ as in Fig. 10. The strong similarity of the uncertainty bands shown in Figs 10 and 11 suggests that the 

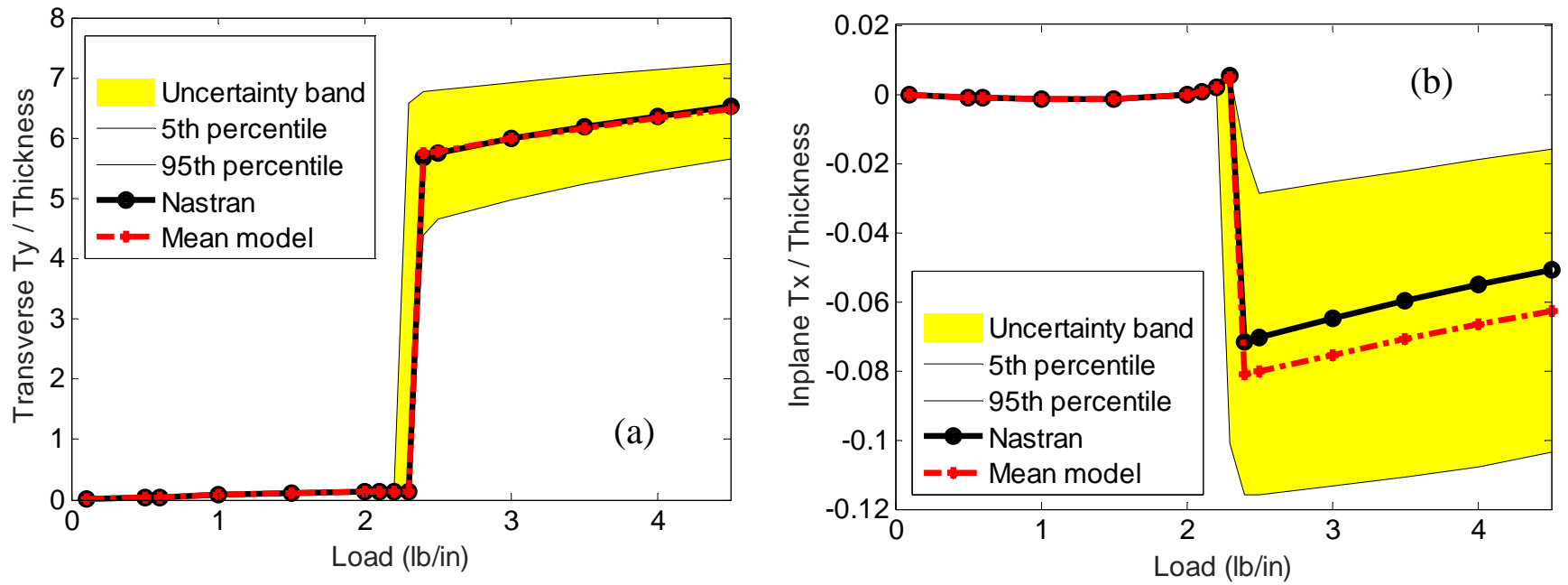

Figure 11. Static displacement of the clamped-clamped curved beam under a uniform loading. (a) Transverse and (b) inplane displacements (in percent of span) of a beam quarter point vs. load. Mean and uncertain models, approach of section 5.5.
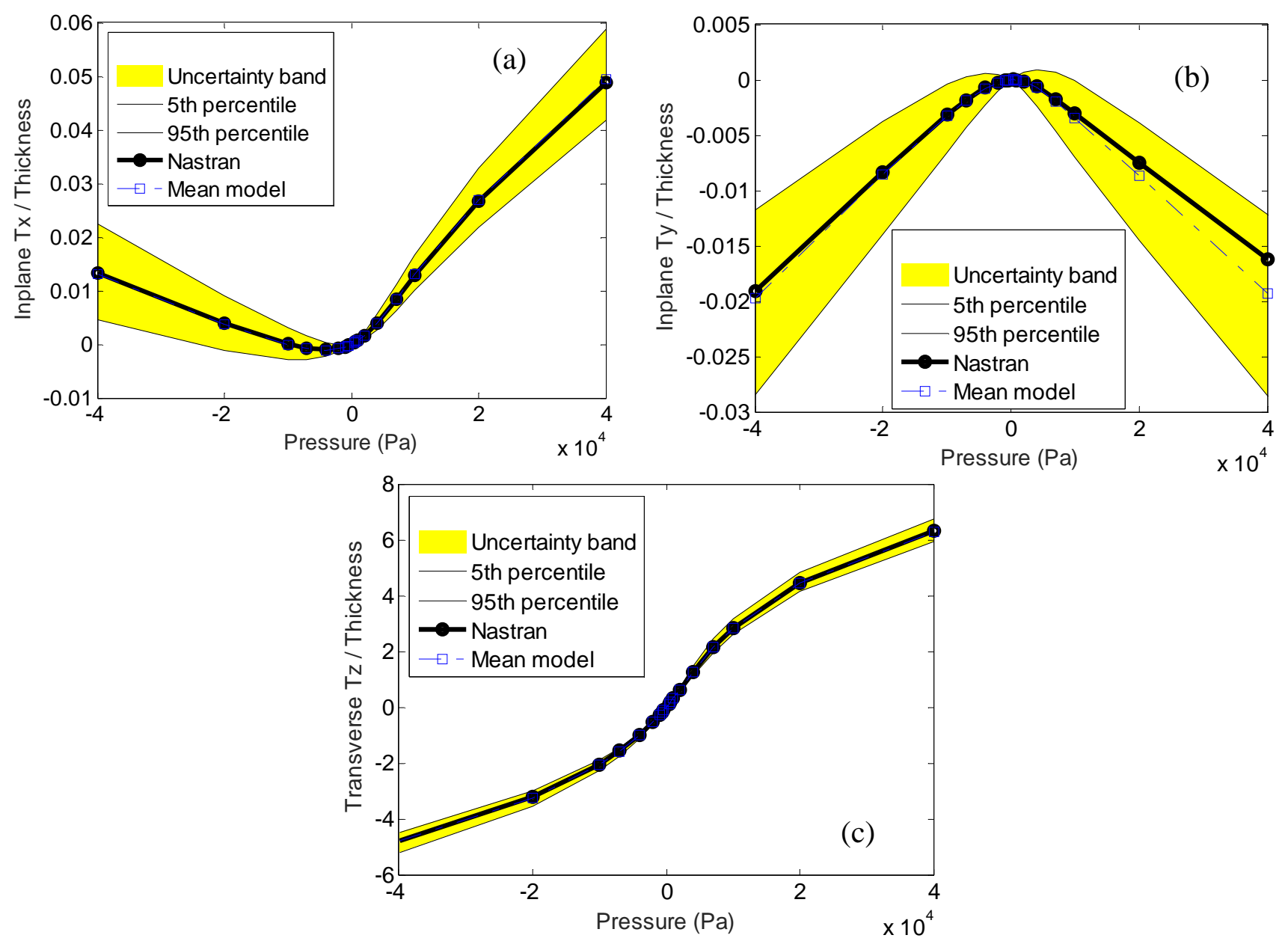

Figure 12. Static displacement of the orthogrid panel under a uniform loading. (a) Inplane $x$, (b) inplane $y$, and (c) transverse $z$ displacements at a quarter point divided by the panel thickness vs. load. Mean and uncertain models, approach of section 5.5. 
introduction of uncertainty on the largest positive definite block $\boldsymbol{K}_{11}$ is an appropriate strategy that is additionally simpler. Another validation was performed on the orthogrid panel of Fig. 7, see Fig. 12 for the results corresponding to $\delta=0.28$. While the uncertainty band is visibly wider than in Figs 8 , it exhibits the same features again supporting the applicability of the approach of section 5.5.

\section{SUMMARY}

The focus of the present investigation was on resolving two key challenges encountered in the introduction of uncertainty in reduced order models of the nonlinear geometric response following an approach proposed earlier by the authors . The first of these challenges was the indeterminacy of the mapping of the nonlinear stiffness coefficients identified from the finite element model, $\bar{K}_{i j l}^{(2)}$ and $\bar{K}_{i j l p}^{(3)}$, to those suitable for the uncertainty analysis, i.e., $\hat{K}_{m n p}^{(2)}$ and $K_{i j l p}^{(3)}$, see Eqs (6),(7), and (19). The second challenge was that the matrix $\overline{\boldsymbol{K}}_{B}$ constructed with these coefficients (and the linear stiffness ones) is not positive definite as showed in the original paper because of differences in nonlinear finite element modeling between the commercial software and the theoretical developments. These challenges were jointly addressed. First, the indeterminacy was resolved by selecting the coefficients to maximize $\overline{\boldsymbol{K}}_{B}$ while satisfying the constraints of Eqs (6), (7), and (19). In general, the resulting matrix $\overline{\boldsymbol{K}}_{B}$ was found not positive definite but a strategy was devised to modify it in a minimal manner to achieve this property. In most of the cases investigated, this modification did not significantly change the predictions of the mean response and thus the uncertainty modeling could proceed from it. In one example, a clamped-clamped curved beam undergoing snap throughs, the changes of mean were large enough. For such situations, a modification of the uncertainty modeling was proposed that handles the non positive definite matrix $\overline{\boldsymbol{K}}_{B}$ with a $\boldsymbol{L} \mathbf{D} \boldsymbol{L}^{\boldsymbol{T}}$ decomposition vs. a Cholesky one. A final alternative to the above modifications was also proposed in which the uncertainty is only introduced on the largest block of the $\overline{\boldsymbol{K}}_{B}$ that is positive definite.

The above methods were applied to 4 different structural models of various complexity and the uncertain response to static loading was determined. These efforts first demonstrated the broad applicability of the above methodology but they also suggested that strong nonlinear features of the response, such as inplane displacements due to transverse loading and post-snap through behavior, are particularly sensitive to uncertainty.

\section{ACKNOWLEDGEMENTS}

The financial support of this investigation by the Air Force Office of Scientific Research grant FA9550-16-1-0021 with Dr. Jaimie Tiley as monitor is gratefully acknowledged. The authors wish to thank the Structural Science Center and specifically Drs R. Gordon and J.J. Hollkamp for the use of the code Bobtran which was used for some of the results in Figure 2.

\section{REFERENCES}

1. Soize, C., (2012). Stochastic Models of Uncertainties in Computational Mechanics. American Society of Civil Engineers.

2. Soize, C., Uncertainty Quantification: An Accelerated Course with Advanced Applications in Computational Engineering, Springer-Verlag, 2017. 
3. Mignolet, M.P., Przekop, A., Rizzi, S.A, and Spottswood, S.M., (2013). A Review of Indirect/NonIntrusive Reduced Order Modeling of Nonlinear Geometric Structures, Journal of Sound and Vibration, 332, 2437-2460.

4. Muravyov, A.A., and Rizzi, S.A., (2003). Determination of Nonlinear Stiffness with Application to Random Vibration of Geometrically Nonlinear Structures, Computers and Structures, 81, 1513-1523.

5. Perez, R.A., Wang, X.Q., and Mignolet, M.P., (2014). Non-Intrusive Structural Dynamic Reduced Order Modeling for Large Deformations: Enhancements for Complex Structures, Journal of Computational and Nonlinear Dynamics, 9, 031008.

6. Capiez-Lernout, E., Soize, C., and Mignolet, M.P., (2014). Post-buckling nonlinear static and dynamical analyses of uncertain cylindrical shells and experimental validation, Computer Methods in Applied Mechanics and Engineering 271, 210-230.

7. Capiez-Lernout, E., Soize, C., and Mignolet, M.P., (2012). Computational Stochastic Statics of an Uncertain Curved Structure with Geometrical Nonlinearity in Three-Dimensional Elasticity, Computational Mechanics 49, 87-97.

8. Capiez-Lernout, E., and Soize, C., (2015). Uncertainty Quantification for an Industrial Mistuned Bladed Disk with Geometrical Nonlinearities, Proceeding of the ASME Turbo Expo 2015, Montreal, Quebec, Canada, June 15-19. Paper No. GT2015-42471.

9. Capiez-Lernout, E., and Soize, C., (2017). An Improvement of the Uncertainty Quantification in Computational Structural Dynamics with Nonlinear Geometrical Effects, International Journal for Uncertainty Quantification 7, 83-98.

10. Mignolet, M.P., and Soize, C., (2008). Stochastic Reduced Order Models for Uncertain Geometrically Nonlinear Dynamical Systems, Computer Methods in Applied Mechanics and Engineering 197, 3951-3963.

11. Wang, X.Q., Perez, R., Mignolet, M.P., Capillon, R., and Soize, C., (2013). Nonlinear Reduced Order Modeling of Complex Wing Models, Proceedings of the 54th Structures, Structural Dynamics and Materials Conference, Boston, Massachusetts, , Apr. 8-11, AIAA Paper AIAA-2013-1520.

12. Kim, K., Radu, A.G., Wang, X.Q., and Mignolet, M.P., (2013). Nonlinear Reduced Order Modeling of Isotropic and Functionally Graded Plates, International Journal of Non-Linear Mechanics, 49, 100-110.

13. Dumir, P.C., and Bhaskar, A., (1988). Some Erroneous Finite Element Formulations of Non-Linear Vibrations of Beams and Plates, Journal of Sound and Vibration, 123(3), 517-527.

14. Morse, H., and Feshbach, P.M., (1953). Methods of Theoretical Physics, McGraw-Hill.

15. Golub, G.H., and van Loan, C.F., (1996). Matrix Computations, 3rd edition, Johns Hopkins University Press.

16. Kim, K., Khanna, V., Wang, X.Q., and Mignolet, M.P., (2009), Nonlinear Reduced Order Modeling of Flat Cantilevered Structures, Proceedings of the 50th Structures, Structural Dynamics, and Materials Conference, Palm Springs, California, May 4-7. AIAA Paper AIAA-2009-2492.

17. Gogulapati, A., Brouwer, K., Wang, X.Q., Murthy, R., MacNamara, J.J., and Mignolet, M.P., (2017). Full and Reduced Order Aerothermoelastic Modeling of Built-Up Aerospace Panels in HighSpeed Flows, Proceedings of the 58th Structures, Structural Dynamics, and Materials Conference, AIAA SciTech, Grapevine, Texas, Jan. 9-13. AIAA Paper AIAA 2017-0180. 